\title{
Qualité de la viande de dromadaire dans les abattoirs de Ouargla en Algérie. II. Contamination bactérienne superficielle des carcasses
}

\author{
A. Benaissa ${ }^{1} *$ A. Ould El Hadj Khelil ${ }^{1}$ A. Adamou ${ }^{1}$ \\ B. Babelhadj ${ }^{1}$ M. Hammoudi ${ }^{1}$ A. Riad ${ }^{1}$
}

\begin{abstract}
Mots-clés
Camelus dromedarius - Viande de chameau - Abattoir - Carcasse Contamination biologique - Algérie.
\end{abstract}

\begin{abstract}
Résumé
Dans le but d'apprécier la qualité microbiologique de la viande de dromadaire produite à l'abattoir de la wilaya de Ouargla en Algérie, la contamination superficielle d'origine bactérienne de 60 carcasses camelines a été évaluée. Les prélèvements ont été réalisés par écouvillonnage de trois sites (cuisse, flanc et épaule). Les échantillons ont été prélevés dans un intervalle d'une heure après l'abattage de l'animal, après le dépouillement, à la fin de l'éviscération et avant l'inspection post mortem. Les taux de germes ont varié en fonction des carcasses et des sites de prélèvement. La flore prédominante a été la flore aérobie mésophile totale, dont le niveau de contamination a été de 2,8 log UFC/ $\mathrm{cm}^{2}$, représentant 25 p. 100 de la flore dénombrée, suivie par les entérobactéries avec 2,4 log UFC/ $\mathrm{cm}^{2}$ (21 p. 100), les coliformes totaux avec 2,2 log UFC/cm² (20 p. 100), les coliformes fécaux avec 2,0 log UFC/ $\mathrm{cm}^{2}$ (18 p. 100), et les staphylocoques avec $1,8 \mathrm{log} \mathrm{UFC} / \mathrm{cm}^{2}$ (16 p. 100). La présence de souches de salmonelles et d'Escherichia coli a été détectée sur toutes les zones prélevées de la carcasse. La viande cameline de l'abattoir de Ouargla a présenté un niveau de contamination élevé par les germes pathogènes (salmonelles et staphylocoques). Une contamination fécale $(E$. coli) élevée a également été observée. De bonnes pratiques d'hygiène durant la phase d'abattage doivent être mises en œuvre afin de diminuer le niveau de contamination.
\end{abstract}

\section{INTRODUCTION}

Bien que la consommation de viande cameline soit très faible à l'échelle nationale, elle est importante dans les régions sahariennes car les camelins représentent 33 p. 100 de l'ensemble des abattages en viande rouge et cette proportion est en progression constante (1). L'abattage des animaux dans les abattoirs constitue une garantie de qualité pour les viandes. Les animaux et les denrées y subissent une inspection sanitaire permanente permettant de dépister des zoonoses et de retirer des circuits de consommation

\footnotetext{
1. Laboratoire de protection des écosystèmes des zones arides et semi-arides, Université Kasdi Merbah, Ouargla 30000, Algérie.

* Auteur pour la correspondance

Tél. : +213772 213516 ; e-mail : benaissaatika@gmail.com
}

humaine des viandes présentant des lésions préjudiciables à leur qualité sanitaire.

Toutefois, l'abattoir représente un lieu où le risque de contamination superficielle des viandes n'est pas négligeable. En effet, les manipulations non hygiéniques pendant l'abattage et la préparation des carcasses conduisent à de fréquentes contaminations superficielles qui peuvent affecter la santé du consommateur et la qualité de la viande (altération organoleptique et sanitaire) (7). Il a été estimé que 80 à 90 p. 100 de la microflore des viandes parvenant aux consommateurs résultait de contaminations croisées survenant à l'abattoir (17). De fait, les étapes de l'abattage, comme le dépouillement et l'éviscération, représentent des moments sensibles pour la contamination microbienne des carcasses. Une grande partie de ces germes sont des saprophytes de la peau et du tube digestif de l'animal, provoquant des altérations possibles de 
la carcasse, comme le poissage, une altération superficielle des carcasses, pouvant aller jusqu'à la putréfaction, c'est-à-dire une altération majeure des viandes si les conditions de conservation au froid ne sont pas respectées. Une fois contaminée, la viande peut être le siège d'une prolifération microbienne car elle constitue un excellent milieu de croissance pour un grand nombre d'espèces bactériennes. Ces contaminations sont souvent indécelables lors d'une simple inspection sanitaire post mortem car il s'agit de contaminations de très petites tailles. Néanmoins, toute souillure visuelle (matière fécale, contenu digestif, souillure environnementale) est une source importante de contamination bactérienne.

Ainsi, comme le montrent plusieurs études, la viande, avec ses dérivés, est très souvent à l'origine des intoxications alimentaires transmises à l'homme : ils représentent 70 p. 100 des cas d'intoxications alimentaires (16). Les maladies infectieuses d'origine alimentaire (ou les toxi-infections alimentaires collectives [TIAC]) sont souvent liées à des défauts d'hygiène et peuvent être très graves (2), mettant principalement en cause les bactéries salmonelles, Escherichia coli entérotoxinogène et staphylocoques à coagulase positive. L'objectif de cette étude a été d'apprécier la qualité microbiologique de la viande cameline produite à l'abattoir de la wilaya de Ouargla en Algérie, en évaluant la contamination superficielle d'origine bactérienne des carcasses de dromadaire.

\section{MATERIEL ET METHODES}

\section{Abattoir de Ouargla}

L'abattoir communal de Ouargla a été sélectionné car il est l'un des plus importants abattoirs d'Algérie pour la production de viande rouge (bovine, ovine, caprine et cameline), estimée à 2000 tonnes par an. Il s'étend sur $85000 \mathrm{~m}^{2}$ (Direction des services agricoles, 2013) et se compose de trois aires de repos, l'une pour les camelins et les deux autres pour les bovins, les ovins et les caprins, et de six salles d'abattage et d'éviscération.

\section{Procédure d'abattage et d'éviscération du dromadaire}

Après repos et diète hydrique, les dromadaires sont soumis à une inspection ante mortem afin de repérer et d'éliminer de la chaîne d'abattage les animaux malades. Ensuite la saignée est l'opération la plus délicate, le dromadaire est mis en position sterno-abdominale (contention), orienté vers la Mecque selon le rituel islamique. L'encolure est repliée le long du corps sur le flanc gauche et l'animal est égorgé (11). Le dépouillement commence après l'ablation de la tête et du cou. Une incision est pratiquée le long de la ligne dorsolombaire et la peau est repliée vers le ventre. La région du thorax et de l'abdomen est dépouillée avant les membres. La bosse est retirée et le cuir arraché. Le dépouillement et l'éviscération du dromadaire se font sur le sol. La cavité abdominale est ouverte. Les viscères abdominaux sont retirés, puis le diaphragme sectionné et les viscères thoraciques sont enlevés.

\section{Protocole expérimental}

Pour apprécier la qualité hygiénique des carcasses, la recherche et le dénombrement des bactéries indicatrices de contamination fécale ou de défaut d'hygiène, et de certains germes pathogènes, comme les salmonelles et les staphylocoques à coagulase positive (22), sont réalisés. Les prélèvements ont été mis en œuvre par méthode non destructive (écouvillon de gaze et eau peptonée tamponnée comme diluant) $(13,18,19)$. Une surface délimitée de $100 \mathrm{~cm}^{2}$ a été frottée pendant au moins 20 secondes, verticalement, horizontalement et en diagonale, en appliquant une pression aussi forte que possible pour prélever les germes éventuellement présents (Journal officiel des Communautés européennes, 2001). Pour chaque carcasse, trois zones ont été écouvillonnées correspondant à une surface totale de $300 \mathrm{~cm}^{2}$ (6). Les prélèvements ont été réalisés deux jours par semaine. Les jours de l'échantillonnage ont varié d'une semaine à l'autre (du lundi au samedi) afin que les résultats soient représentatifs de toute la semaine. Les échantillons ont été prélevés dans un intervalle d'une heure après l'abattage, après le dépouillement, à la fin de l'éviscération et avant l'inspection post mortem. Au total, 180 échantillons provenant de 60 dromadaires abattus ont été analysés. Un échantillon par carcasse a consisté en trois prélèvements sur trois sites différents (cuisse, flanc et épaule). Les échantillons ont été transportés rapidement dans une glacière au laboratoire de l’Université Kasdi Merbah de Ouargla.

\section{Caractérisation microbiologique des surfaces étudiées}

La préparation de la solution mère et des dilutions décimales a été réalisée selon la norme française NF V-057-2. Le milieu de culture utilisé pour le dénombrement de la flore aérobie mésophile totale a été le dénombrement sur plaque de gélose, selon la norme ISO 4833. Les coliformes fécaux ont été dénombrés sur gélose lactosée biliée au cristal violet et au rouge neutre (VRBL), selon la norme NF V08-017, avec incubation des boîtes de Petri ensemencées à $44{ }^{\circ} \mathrm{C}$. Pour le dénombrement des coliformes totaux, le milieu VRBL a été utilisé, et les boîtes de Petri ensemencées et incubées à $37^{\circ} \mathrm{C}$. Pour les entérobactéries, le milieu gélosé sélectif VRBG a été ensemencé dans la masse à $37^{\circ} \mathrm{C}$. Les staphylocoques ont été dénombrés sur milieu de Chapman (9). La recherche des salmonelles a été réalisée selon la norme NF V08-052. Un pré-enrichissement de $1 \mathrm{ml}$ de la solution correspondant au prélèvement a été porté dans un tube contenant $9 \mathrm{ml}$ d'eau peptonée tamponnée stérile, après incubation à $37^{\circ} \mathrm{C}$ pendant 16 à $20 \mathrm{~h}$. Il a été suivi par un enrichissement dans du bouillon Rappaport Vassiliadis soja (RVS), soit 0,1 ml de la solution de pré-enrichissement dans $10 \mathrm{ml}$ de milieu RVS, avec incubation à $37{ }^{\circ} \mathrm{C}$ pendant 18 à $24 \mathrm{~h}$. Un isolement a été réalisé par ensemencement en stries à la surface du milieu Hektoen à partir du bouillon d'enrichissement. Les boîtes ont été incubées à $37^{\circ} \mathrm{C}$ pendant 18 à $24 \mathrm{~h}$, parfois même pendant $48 \mathrm{~h}$, en cas d'absence de colonies caractéristiques (colonies bleues) après la première lecture (21). Les résultats des dénombrements ont été exprimés en log du nombre d'unités formant colonies (UFC) $/ \mathrm{cm}^{2}$.

L'identification biochimique d'E. coli a été réalisée à partir des colonies présentes sur milieu VRBL incubé à $44{ }^{\circ} \mathrm{C}$ (triple sucre fer [TSI], citrate de Simmons, production de gaz, uréase, production d'indole et production d'hydrogène sulfuré). La détermination de la mobilité a été obtenue par l'utilisation du mannitol mobilité.

\section{RESULTATS}

Le tableau I montre la flore bactérienne par site de prélèvement. Le dénombrement des germes de la contamination globale des carcasses camelines a révélé des moyennes de contamination par la flore aérobie mésophile totale qui constituait la flore prédominante ( 25 p. 100 de la flore bactérienne globale dénombrée) de $2,8 \pm 0,27 \log \mathrm{UFC} / \mathrm{cm}^{2}$, suivie par les entérobactéries avec 2,4 $\pm 0,28 \mathrm{log} \mathrm{UFC} / \mathrm{cm}^{2}$ (21 p. 100), les coliformes totaux avec 2,2 $\pm 0,26 \log \mathrm{UFC} / \mathrm{cm}^{2}$ (20 p. 100), les coliformes fécaux avec 2,0 $\pm 0,22 \mathrm{log} \mathrm{UFC} / \mathrm{cm}^{2}$ (18 p. 100), et les staphylocoques avec 1,8 $\pm 0,44 \log \mathrm{UFC} / \mathrm{cm}^{2}$ (16 p. 100).

La flore aérobie mésophile totale a été prédominante au niveau de la contamination globale des carcasses comme au niveau de chaque site. La surface des cuisses a été la zone la plus contaminée par cette flore, suivie par le flanc et l'épaule. Les 


\section{Tableau I}

Flore bactérienne prélevée sur de la viande de dromadaires à l'abattoir de Ouargla en Algérie

\begin{tabular}{|c|c|c|c|c|c|}
\hline \multirow[t]{2}{*}{ Dénombrement (log UFC/cm²) } & \multirow[t]{2}{*}{ Paramètre calculé } & \multicolumn{4}{|c|}{ Site de prélèvement } \\
\hline & & Cuisse & Epaule & Flanc & Carcasse \\
\hline \multirow[t]{3}{*}{ Flore aérobie mésophile totale } & Nb. carcasses & 60 & 60 & 60 & 60 \\
\hline & Moyenne & 2,9 & 2,8 & 2,7 & 2,8 \\
\hline & Ecart-type & 0,39 & 0,16 & 0,27 & 0,27 \\
\hline \multirow[t]{3}{*}{ Entérobactéries } & Nb. carcasses & 48 & 48 & 48 & 48 \\
\hline & Moyenne & 2,4 & 2,3 & 2,3 & 2,4 \\
\hline & Ecart-type & 0,34 & 0,27 & 0,24 & 0,28 \\
\hline \multirow[t]{3}{*}{ Coliformes totaux } & Nb. carcasses & 48 & 48 & 48 & 48 \\
\hline & Moyenne & 2,2 & 2,1 & 2,2 & 2,2 \\
\hline & Ecart-type & 0,31 & 0,24 & 0,23 & 0,26 \\
\hline \multirow[t]{3}{*}{ Coliformes fécaux } & Nb. carcasses & 49 & 49 & 49 & 49 \\
\hline & Moyenne & 2,0 & 1,9 & 2,1 & 2,0 \\
\hline & Ecart-type & 0.30 & 0.25 & 0.11 & 0.22 \\
\hline \multirow[t]{3}{*}{ Staphylocoques } & Nb. carcasses & 53 & 53 & 53 & 53 \\
\hline & Moyenne & 1,9 & 1,9 & 1,7 & 1,8 \\
\hline & Ecart-type & 0,48 & 0,48 & 0,38 & 0,44 \\
\hline \multirow[t]{2}{*}{ Salmonelles } & Nb. carcasses & 39 & 39 & 39 & 39 \\
\hline & Présence (\%) & 100 & 100 & 100 & 100 \\
\hline \multirow[t]{2}{*}{ Escherichia coli } & Nb. carcasses & 32 & 32 & 32 & 32 \\
\hline & Présence (\%) & 100 & 100 & 100 & 100 \\
\hline
\end{tabular}

entérobactéries sont apparues en plus grand nombre sur la cuisse, suivie par l'épaule et le flanc. Globalement, cuisse et flanc ont été les zones les plus contaminées par les coliformes totaux, suivis par l'épaule. La même constatation a été faite pour les staphylocoques qui ont été plus nombreux sur la cuisse, suivie par le flanc, puis l'épaule. Cependant cette variabilité interzone n'a pas été statistiquement significative. Les salmonelles ont affecté tous les sites étudiés.

E. coli a été identifié à partir des colonies des coliformes fécaux (tableau II).

\section{DISCUSSION}

Parmi les germes dénombrés, la flore aérobie mésophile, les entérobactéries et les salmonelles ont été les trois indicateurs principaux de l'hygiène du procédé d'abattage (Commission européenne, 2005). De même, E. coli a renseigné sur les conditions de l'abattage (5). Les résultats obtenus ont été comparés aux données disponibles de quelques abattoirs algériens.

La flore aérobie mésophile totale est une flore indicatrice des bonnes pratiques d'hygiène. Cette flore se développe à température ambiante. Sa prédominance a déjà été signalée par Hamad (14) qui rapporte un taux de contamination moyen de 1,79 $\log \mathrm{UFC} / \mathrm{cm}^{2}$, et par Benaissa (4) qui indique une moyenne de 3,02 log UFC/g de viande cameline. Cette prédominance a été observée aussi bien

\section{Tableau II}

Identification d'Escherichia coli prélevé sur de la viande de dromadaires à l'abattoir de Ouargla en Algérie par les tests biochimiques et de détermination de la mobilité

\begin{tabular}{lc} 
Caractère d'identification & E. coli \\
\hline Fermentation de glucose & + \\
Fermentation de lactose & + \\
Production de gaz & + \\
Production $\mathrm{d}^{\prime} \mathrm{H}_{2} \mathrm{~S}$ & - \\
Citrate de Simmons & - \\
Indole & + \\
Manitol & + \\
Uréase & - \\
Mobilité & +
\end{tabular}

+ : présence ; - : absence

au niveau de la carcasse globale que sur chacun des trois sites échantillonnés. La valeur de 2,8 $\pm 0,27 \log \mathrm{UFC} / \mathrm{cm}^{2}$ a été supérieure à celle relevée à l'abattoir d'El Oued (14) sur des carcasses camelines, attestant de conditions d'hygiène plus défectueuses dans l'abattoir de Ouargla. Cette valeur a cependant été inférieure 
à celles rapportées dans les abattoirs d'Alger $\left(4,48 \log \mathrm{UFC} / \mathrm{cm}^{2}\right)$ (19), de Constantine (5,34 log UFC/ $\left.\mathrm{cm}^{2}\right)$ (10) et de Tiaret (3,17 log $\left.\mathrm{UFC} / \mathrm{cm}^{2}\right)(15)$ sur des carcasses bovines. A Ouargla, Benaissa (4) a enregistré des valeurs plus élevées (3,02 log UFC/g en moyenne) sur la viande cameline avec une méthode de prélèvement destructive basée sur l'utilisation d'un emporte-pièce. Cette méthode d'excision induit généralement des valeurs plus élevées (23).

Le taux de contamination par les entérobactéries de 2,4 log UFC/ $\mathrm{cm}^{2}$ a confirmé la présence d'une contamination d'origine fécale liée au non-respect des règles d'hygiène et pouvant avoir eu pour origine le tube digestif ou le sol contaminé lors de l'éviscération. Ces résultats sont proches de ceux de Benaissa (4) qui rapporte une valeur moyenne de 2,27 log UFC/g pour cette viande. A l'inverse, Hamad (14) n'a dénombré que $0,84 \log \mathrm{UFC} / \mathrm{cm}^{2}$ à l'abattoir d'El-Oued. La cuisse a semblé héberger des charges microbiennes plus élevées par rapport aux deux autres sites étudiés, notamment l'épaule, plus faiblement contaminée, contrairement aux résultats obtenus par Hamad (14), et El-Hadef et Hokki (10) qui rapportent des charges microbiennes plus élevées respectivement de l'épaule $\left(2,13 \log \mathrm{UFC} / \mathrm{cm}^{2}\right)$ et du flanc $\left(1,21 \log \mathrm{UFC} / \mathrm{cm}^{2}\right)$.

Les coliformes représentent une portion assez considérable de la flore aérobie mésophile totale (3). Les taux élevés de coliformes totaux et fécaux (respectivement de 2,2 et $2 \log \mathrm{UFC} / \mathrm{cm}^{2}$ ) dans nos échantillons ont été proches de ceux signalés par Benaissa (2,04 log UFC/g coliformes fécaux) avec la technique d'excision (4). Ils ont cependant été supérieurs à ceux relevés par Hamad sur des carcasses camelines $\left(0,50 \log \mathrm{UFC} / \mathrm{cm}^{2}\right)$ à El Oued (14), et par Nouichi et Hamdi sur des carcasses bovines (respectivement 2,92 et 2,6 $\log \mathrm{UFC} / \mathrm{cm}^{2}$ pour les coliformes totaux et fécaux) à Alger (19).

Ces flores sont révélatrices de conditions d'hygiène et de manipulations des carcasses insuffisantes. Elles sont particulièrement indicatrices de contaminations fécales et par conséquent liées à des négligences survenues lors du dépouillement et de l'éviscération, ces étapes de l'abattage étant considérées comme étant les sources de contamination des carcasses les plus importantes, ou de comportements non hygiéniques des manipulateurs. En effet, les coliformes sont des bactéries saprophytes du tube digestif de l'homme et des animaux (3). La présence des staphylocoques indique une contamination à partir de la tête de l'animal (oreilles, amygdales, gorge) ou résultant de la manipulation des carcasses par un personnel pouvant être atteint de rhinopharyngite à staphylocoques, d'angine ou de lésion cutanée infectée aux mains (9).

La présence de salmonelles sur toutes les zones prélevées (100 p. 100) a été supérieure aux valeurs rapportées par Hammoudi et coll. (21 p. 100) (15), et Nouichi et Hamdi (10 p. 100) (20) sur des carcasses bovines. Leur provenance pouvait être diverse, comme les réservoirs gastro-intestinaux, le cuir, les mains des opérateurs et l'équipement (20). La présence d'E. coli atteste d'une contamination fécale, indiquant à nouveau une hygiène défectueuse et des procédures d'abattage non conformes (20).

Le contrôle microbiologique dans les abattoirs algériens n'est pas obligatoire. De plus, les normes nationales ne mentionnent ni le type de prélèvement à pratiquer, ni la zone à prélever. Celles-ci devraient être révisées, notamment en concordance avec le règlement européen (CE). Néanmoins, le respect des bonnes pratiques d'hygiène lors de l'abattage et la réalisation de cet abattage dans des locaux et avec des équipements adaptés s'avèrent des éléments clés pour fournir une viande de qualité microbiologique satisfaisante. Tout contact des dromadaires abattus avec le sol devrait être évité. L'usage d'équipements spécialisés pourrait être recommandé, notamment la suspension de la carcasse lors du dépouillement et de l'éviscération (12). Le projet de construction d'abattoirs industriels peut s'avérer avantageux pour le consommateur désirant un produit de meilleure qualité bactériologique. Un tel produit pourrait dès lors être conservé plus long temps et consommé sans danger sanitaire.

\section{CONCLUSION}

La viande demeure une source essentielle de protéines pour l'homme. Cependant, son importance sanitaire et hygiénique, et son caractère périssable ont incité les pouvoirs publics à mettre en place des structures d'abattage contrôlées (les abattoirs). Les résultats des analyses microbiologiques réalisées à partir de différents échantillons prélevés à la surface des carcasses camelines à l'abattoir de Ouargla en Algérie montrent que le niveau de contamination par la majorité des germes dénombrés a été élevé, avec notamment la détection de germes pathogènes pour l'homme, comme les salmonelles, les staphylocoques et $E$. coli. Le strict respect des bonnes pratiques d'hygiène dans les abattoirs est donc essentiel pour la prévention de la contamination microbienne des carcasses, en vue de préserver au mieux la qualité des viandes, avec comme conséquence la protection de la santé du consommateur.

Des chaînes d'abattage permettant la continuité du processus d'abattage et évitant tout contact des carcasses avec le sol et les contaminations croisées sont recommandées. Tout processus d'abattage devrait être complété par une analyse microbiologique des carcasses sur des prélèvements réalisés de façon aléatoire. La salubrité est estimée par un contrôle vétérinaire post mortem, mais l'innocuité alimentaire de ces carcasses dépend essentiellement de la qualité des pratiques d'abattage et de la préparation de la carcasse, vérifiées par des analyses microbiologiques. La méthode hazard analysis and critical control point (HACCP) doit être mise en place dans les abattoirs algériens afin de prévenir les toxi-infections collectives.

\section{BIBLIOGRAPHIE}

1. ADAMOU A., 2011. Développement de la filière viande cameline pour la sécurité alimentaire des populations du Sahara algérien. In : atelier sous-régional, Effet du changement climatique sur l'élevage et la gestion durable des parcours dans les zones arides et semi-arides du Maghreb, Ouargla, Algérie, 21-24 nov. 2011, p. 82.

2. ARVIEUX C., 1998. Les toxi-infections alimentaires. Digest, 14 : 4-16.

3. BASEL M.R., RICHTER E.R., BANWART G.J., 1983. Monitoring microbial numbers in food by density centrifugation. Appl. Env. Microbiol., 45: 1156-1159.

4. BENAISSA A., 2011. Etude de la qualité microbiologique des viandes cameline et ovine conservées selon différents modes. Mém. Magister Microbiol. Appl., Université Kasdi Merbah, Ouargla, Algérie, p. 43-54.

5. CARTIER P., 1990. Méthodologie de contrôle de la qualité hygiénique d'un avant de bovins. Viandes Prod. Carnés, 11 : 215-216.

6. CARTIER P., 1993. Importance, origine et mode d'appréciation de la contamination salmonellique de la carcasse des bovins. Examen de 222 vaches de réforme. Viandes Prod. Carnés, 14 : 35-38.

7. DENNAI N., KARRATI B., EL YACHIOUI M., 2000. Une microbiologie fluctuante. Viandes Prod. Carnés, 21 : 191-196.

8. DENNAI N., KHARRATI B., EL YACHIOUI M., 2001. Appréciation de la qualité microbiologique des carcasses de bovins fraîchement abattus. Ann. Méd. Vét., $145: 270-274$.

9. DESMARCHELIER P.M., HIGGS M.G., MILLS L., SULLIVAN A.M., VANDERLINDE P.B., 1999. Incidence of coagulase positive staphylococcus on beef carcasses in three Australian abattoirs. Int. J. Food Microbiol., 47: 221-229. DOI: 10.1016/S0168-1605(99)00018-5 
10. EL-HADEF EL OKKI S., EL-GROUD R., KENANA H., QUESSY S., 2005. Evaluation de la contamination superficielle des carcasses bovines et ovines provenant de l'abattoir municipal de Constantine en Algérie. Can. Vet. J., 46 : 638-640.

11. FAYE B., 1997. Guide de l'élevage du dromadaire. Libourne, France, Sanofi, $126 \mathrm{p}$.

12. FAYE B., ABDELHADI O., RAIYMBEK G., KADIM I., HOCQUETTE J.F., 2013. La production de la viande de chameau : état des connaissances, situation actuelle et perspectives. Prod. Anim., 26 : $247-$ 258

13. FLISS I., SIMARD R.E., ETTRIKI A., 1991. Comparison of three sampling techniques for microbiological analysis of meat surfaces. J. Food Sci., 56: 249-252. DOI: 10.1111/j.1365-2621.1991.tb08021.x

14. HAMAD B., 2009. Contribution à l'étude de la contamination superficielle bactérienne et fongique des carcasses camelines au niveau de I'abattoir d'El-Oued. Mém. Magister Méd. Vét., Université de Constantine, Algérie, p. 29-30.

15. HAMMOUDI A., BOUSMAHA F. BOUZID R., AGGAD $\mathrm{H}$., SAEGERMAN C., 2013. Evaluation de la contamination bactérienne superficielle des carcasses bovines dans un abattoir algérien. J. Anim. Plant Sci., 19 : 2901-2907.

16. HOBBS B.C., GILBERT R.J., 1978. Food poisoning and food hygiene, 4th Edn. Westport, CT, USA, Food and Nutrition Press, 366 p.

17. JOUVE J.L., 1990. Microbiologie alimentaire et filière des viandes. Viandes Prod. Carnés, 11 : 207-213.
18. KARIB H., YANGUELA J., BLANCO D., CARRAMINANA J.J., HERREA A., 1993. Appréciation de la qualité microbiologique des carcasses et des viscères d'agneaux fraîchement abattus. Viandes Prod. Carnés, $11:$ 118-129

19. KHALIFA A.H., 1985. Origine des contaminations superficielles des carcasses de bovins à l'abattoir, techniques de prélèvement. Mém. DEA, Ecole nationale vétérinaire de Lyon, France, p. 4-36.

20. NOUICHI S., HAMDI T.M., 2009. Superficial bacteria contamination of ovine and bovine carcasses at El-Harrach slaughterhouse (Algeria). Eur. J. Sci. Res., 38: 474-485.

21. RODIER J., BAZIN C., CHANBON P., BROUTIN J.P., CHAMPSAUR H., RODI L., 1996. L'analyse de l'eau : eaux naturelles, eaux résiduaires et eaux de mer, $8^{\mathrm{e}}$ édn. Paris, France, Dunod, p. 1383.

22. WARE L.M., KAIN M.L., SOFOS J.N., BELK K.E., SMITH C.G. 1999. Comparison of sponging and excising as sampling procedures for microbiological analysis of fresh beef-carcass tissue. J. Food Prot., 62 : 1255-1259.

23. ZWEIFEL C., BALTZER D., STEPHAN R., 2005. Microbiological contamination of cattle and pig carcasses at five abattoirs determined by swab sampling in accordance with EU decision 2001/471/EC. Meat Sci., 69: 559-566. DOI: 10.1016/j.meatsci.2004.10.007

Accepted 18 August 2015; Online publication 30 September 2015

\section{Summary}

Benaissa A., Ould El Hadj Khelil A., Adamou A., Babelhadj B., Hammoudi M., Riad A. Camel meat quality in the slaughterhouse of Ouargla in Algeria. II. Bacterial surface contamination of carcasses

To assess the microbiological quality of camel meat in the slaughterhouse of Ouargla District in Algeria, the bacterial surface contamination of 60 camel carcasses was investigated. Within an hour after slaughter, swabs were used to collect samples on three sites (thigh, flank and shoulder), just after skinning and before postmortem inspection. Germ rates varied depending on the carcasses and sampling sites. The predominant flora was the total aerobic mesophilic flora, whose contamination level was $2.8 \mathrm{log} \mathrm{CFU} / \mathrm{cm}^{2}$ (i.e. $25 \%$ of the flora count), followed by enterobacteria with $2.4 \mathrm{log} \mathrm{CFU} / \mathrm{cm}^{2}$ (21\%), total coliforms with $2.2 \mathrm{log} \mathrm{CFU} / \mathrm{cm}^{2}(20 \%)$, fecal coliforms with $2.0 \log \mathrm{CFU} / \mathrm{cm}^{2}(18 \%)$, and staphylococci with $1.8 \mathrm{log}$ CFU/cm ${ }^{2}$ (16\%). Salmonella and Escherichia coli were detected on all the sampled sites of the carcasses. The camel meat of Ouargla slaughterhouse showed a high level of contamination by pathogenic bacteria (salmonellae and staphylococci). A high fecal contamination (E. coli) was also present. Good hygiene practices need to be implemented at slaughter to reduce contaminations.

Keywords: Camelus dromedarius - Camel meat - Abattoir Carcass - Biological contamination - Pathogenic bacteria Algeria.

\section{Resumen}

Benaissa A., Ould El Hadj Khelil A., Adamou A., Babelhadj B., Hammoudi M., Riad A. Calidad de la carne de dromedario en los mataderos de Ouargla en Argelia. II. Contaminación bacteriana de las carcasas

Con el fin de evaluar la calidad microbiológica de la carne de dromedario producida en el matadero de wilaya de Ouargla en Argelia, se evaluó la contaminación superficial de origen bacteriana en 60 carcasas camelinas. Las muestras fueron realizadas por frotis en tres zonas (muslo, flanco y hombro). Las muestras fueron obtenidas en un intervalo de una hora después del sacrificio y destace del animal, al final de la evisceración y antes de la inspección post mortem. Las tasas de gérmenes variaron según la carcasa y los sitios de muestreo. La flora predominante fue la flora aeróbica mesófila total, cuyo nivel de contaminación fue de 2,8 $\mathrm{log} \mathrm{UFC} / \mathrm{cm}^{2}$, representando $25 \%$ de la flora identificada, seguida por enterobacterias, con 2,4 log UFC/cm ${ }^{2}(21 \%)$, los coliformes con 2,2 log UFC/ $\mathrm{cm}^{2}(20 \%)$, los coliformes fecales con 2,0 log UFC/ $\mathrm{cm}^{2}$ (18\%) y los estafilococos con $1,8 \mathrm{log}$ UFC/cm ${ }^{2}(16 \%)$. La presencia de cepas de Salmonella y de Escherichia coli fue detectada en todas las zonas de muestro de la carcasa. La carne camelina del matadero de Ouargla presentó un nivel de contaminación elevado para los gérmenes patógenos (salmonelas y estafilococos). Una contaminación fecal (E. coli) elevada fue igualmente observada. Buenas prácticas de higiene durante la fase de matanza deben ser establecidas con el fin de disminuir el nivel de contaminación.

Palabras clave: Camelus dromedarius - Carne de camello Matadero - Canal animal - Contaminación biológica Argelia. 
